\title{
Die Zirkumzision im Spannungsfeld zwischen Religionsfreiheit und dem Recht auf körperliche Unversehrtheit
}

\author{
Circumcision Might Cause Conflicts Between Fundamental Rights
}

Autoren

Institute
M. Ritter ${ }^{1}$, J. Schabbeck ${ }^{2}$

${ }^{1}$ Klinik für Urologie, Universitätsmedizin Mannheim

${ }^{2}$ Rechtsanwalt und Fachanwalt für Medizinrecht, Ludwigshafen
Bibliografie

DOI http://dx.doi.org/

10.1055/s-0034-1364958

Online-Publikation: 11.2.2014

Akt Dermatol 2014; 40: 133-136

(c) Georg Thieme Verlag KG

Stuttgart · New York

ISSN 0340-2541

Korrespondenzadresse: PD Dr. med. Manuel Ritter Klinik für Urologie Universitätsmedizin Mannheim Ruprecht-Karls-Universität Heidelberg

Theodor Kutzer Ufer 1-3 68167 Mannheim manuel.ritter@umm.de

\begin{abstract}
Zusammenfassung
$\nabla$

Die Beschneidung der männlichen Vorhaut (Zirkumzision) stellt neben einer medizinisch erforderlichen Maßnahme auch einen bedeutsamen Ritus verschiedener Religionsgemeinschaften und Gesellschaften dar. Die Diskussion wird dabei auf den verschiedensten Ebenen und von vielen Interessensgemeinschaften geführt. Der Artikel beleuchtet einerseits die historischen und medizinischen Indikationen zur Zirkumzision und andererseits die rechtlichen Hintergründe und Grundlagen der aktuellen Diskussion.
\end{abstract}

Hintergrund

$\nabla$

Die Zirkumzision stellt die operative Therapie der Wahl bei der Behandlung einer sekundären Phimose oder nach frustraner konservativer Therapie einer primären Phimose dar. Bei guten Ansprechraten der konservativen Therapie ist die Indikation zur operativen Therapie aus medizinischen Gründen nur selten zu stellen. Historisch betrachtet wurde die Zirkumzision auch in der Fachliteratur bis in die 70er-Jahre als probates Mittel zur Vermeidung oder Reduktion der Masturbation angesehen [1]. Aktuell sind die meisten Zirkumzisionen nicht medizinisch begründet sondern religiös motiviert. Ob bei religiös motivierten operativen Eingriffen bei nicht einwilligungsfähigen Patienten der Tatbestand einer strafbaren Körperverletzung erfüllt ist oder ob die Entscheidung zur Durchführung des Eingriffes durch das Grundrecht auf Religionsfreiheit der Eltern und ihr Recht auf freie Erziehung ihrer Kinder im Rahmen der Personensorge gerechtfertigt sei, war immer wieder Gegenstand intensiver Diskussionen [2] und gipfelte 2012 in einer vielbeachteten Entscheidung des Landgerichts Köln [3], welches von der Strafbarkeit der nicht medizinisch indizierten Zirkumzision ausging. Nachdem die Rechtslage zu dem Thema der nicht medizinisch indizierten Beschneidungen lange Zeit zwar rechtlich diskutiert wurde, allerdings unproblematisch praktiziert wurde, trat aufgrund der Entscheidung des Landgerichts Köln am 28.12.2012 der §1631d des Bürgerlichen Gesetzbuches der Bundesrepublik Deutschland in Kraft. Dieser erlaubt den Eltern in die religiös motivierte Beschneidung eines nicht einwilligungsfähigen Kindes einzuwilligen, wenn die Beschneidung nach den Regeln der ärztlichen Kunst durchgeführt wird.

\section{Phimose}

\section{$\nabla$}

Als Phimose wird die Verengung des Präputiums bezeichnet, die die Retraktion entweder erschwert (relative Phimose) oder unmöglich macht (absolute Phimose). Man unterscheidet die primäre Phimose durch eine Verklebung der Vorhautblätter, die im Säuglingsalter physiologisch auftritt, von der sekundären Phimose als Folge rezidivierender Entzündungen. So ist die Retraktion der Vorhaut im ersten Lebensjahr nur bei etwa 50\% der Jungen möglich, während im 3. Lebensjahr bereits bei $89 \%$ der Jungen die Vorhaut problemlos retrahiert werden kann. Die Inzidenz nimmt mit zunehmendem Alter weiter ab, sodass im Schulalter nur noch bei $8 \%$ eine Phimose besteht. Eine ausgeprägte Phimose kann in seltenen Fällen zu Komplikationen führen. So kann es zu rezidivierenden Harnwegsinfekten und schmerzhaften Balanitiden kommen. Bei forcierter Retraktion des Präputiums kann eine Paraphimose auftreten. Durch die Phimose können obstruktive Miktionsbeschwerden bis hin zum Harnverhalt entstehen.

\section{Diagnostik}

Die Untersuchung zur Erkennung einer Phimose sollte in möglichst entspannter Atmosphäre und im Beisein eines Elternteils erfolgen. Ältere Kin- 
der sollten nach Möglichkeit die Vorhaut selbst soweit zurückziehen, wie es geht. Bei kleineren Kindern kann dies vorsichtig durch einen Elternteil oder den Arzt erfolgen. In jedem Fall sollte nicht forciert versucht werden, die Vorhaut zu retrahieren, um ein Einreißen mit Schmerzen und Narbenbildung zu vermeiden.

\section{Therapie}

Zur Behandlung der Phimose stehen konservative und operative Therapieverfahren zur Verfügung.

Zur konservativen Therapie ist die lokale Applikation mit steroidhaltiger Salbe empfohlen. Dies kann zum Beispiel mit 0,05-0,1\% Betamethasonsalbe $2 \times$ täglich für 30 Tage erfolgen. Die Therapie kann bei nicht ausreichendem Ansprechen für weitere 30 Tage fortgeführt werden. Die Ansprechraten der Therapie liegen je nach Ausprägung der Phimose bei 70-90\%. Die Rezidivraten liegen unter $10 \%$ [4].

Zur operativen Therapie der Phimose steht die radikale oder plastische Zirkumzision zur Verfügung. Die Indikation zur operativen Therapie wird gestellt bei der sekundären Phimose, rezidivierenden Balanitiden oder Harnwegsinfekten, frustraner konservativer Therapie oder beim Erwachsenen aufgrund schmerzhafter Erektionen oder Einrisse der Vorhaut beim Geschlechtsverkehr. Kontraindikationen zur Durchführung des Eingriffs stellen akute Entzündungen, nicht behandelte Gerinnungsstörungen oder eine bestehende Hypospadie vor einer geplanten operativen Therapie dar. Die Zirkumzision sollte bei Kindern in Vollnarkose durchgeführt werden. Zusätzlich sollte bei OP-Ende ein Penis-Wurzelblock mit Lokalanästhetikum durchgeführt werden, da dies postoperative Schmerzen deutlich reduzieren kann. Bei Erwachsenen kann der Eingriff problemlos in Lokalanästhesie mit z.B. $0,1 \mathrm{ml} / \mathrm{kg} \mathrm{KG}$ Bupivacain 0,5\% pro Seite durchgeführt werden.

Bei der radikalen Zirkumzision wird das gesamte innere und äußere Vorhautblatt entfernt, während bei der plastischen Zirkumzision nur der Teil mit der Verengung entfernt wird. Die Komplikationsraten durch die Zirkumzision sind mit ca. $1 \%$ gering. Nachblutungen und Wundinfektionen treten dabei am häufigsten auf. In seltenen Fällen kann es zu Meatus- oder Urethrastenosen, Urethrafisteln oder Sensibilitätsstörungen kommen. Schwerwiegende Komplikationen wie Glansnekrosen sind in Einzelfällen berichtet worden.

\section{Vor- und Nachteile der Zirkumzision \\ $\nabla$}

Die Vor- und Nachteile der Zirkumzision werden insbesondere vor dem Hintergrund der religiös motivierten Beschneidungen intensiv und kontrovers diskutiert. In vielen Bereichen ist die Datenlage nicht eindeutig und lässt Platz für Interpretationen. Der Einfluss der Zirkumzision auf die Sexualität beispielsweise wird besonders kontrovers diskutiert. Verschiedene Studien beschreiben einen negativen Einfluss der Zirkumzision auf die sexuelle Erfüllung der befragten Männer, was auf die ausgeprägte sensible Innervation der Vorhaut und ihre mechanische Schutzfunktion der Glans penis zurückgeführt wird. Durch eine Desensibilisierung der Glans nach der Zirkumzision soll die Empfindsamkeit während des Geschlechtsverkehrs herabgesetzt sein. Zusätzlich sei die sexuelle Erfüllung durch Masturbation nach der Beschneidung reduziert [5]. Andere Arbeiten wiederum belegen den gegenteiligen Effekt und beschreiben eine verbesserte sexuelle Zufriedenheit der Männer und der befragten Partnerinnen durch die Zirkumzision. Dabei seien beschnitte Männer besser in der Lage, die Erektion länger aufrecht zu erhalten, und leiden seltener unter einer Ejaculatio praecox [6]. Insgesamt ist die Frage nicht abschließend geklärt, und es scheint soziokulturelle Unterschiede in Abhängigkeit von den befragten Gruppen zu geben.

Als weiterer Vorteil einer Zirkumzision wird gerne eine bessere Hygiene angeführt. Dieses Argument mag in Regionen mit ausgeprägter Wasserknappheit seine Berechtigung gehabt haben. In der industriellen Welt kann die Indikation zur Zirkumzision jedoch sicher nicht mit der Absicht auf bessere Hygiene gestellt werden. Regelmäßiges Reinigen des Penis mit Retraktion der Vorhaut ist die wesentlich effektivere Maßnahme zur Optimierung der Hygiene in diesem Bereich.

Der Einfluss der Zirkumzision auf sexuell übertragbare Erkrankungen war Gegenstand multipler epidemiologischer Studien.

Bei in Afrika durchgeführten Untersuchungen zeigten sich Hinweise auf eine höhere Rate an sexuell übertragbaren Krankheiten bei nicht beschnittenen Männern. Insbesondere für HIV-Infektionen wurde dieser Zusammenhang gezeigt. Diese Ergebnisse führten zur Einschätzung der WHO, in Ländern mit hoher HIV-Prävalenz und niedriger Rate an beschnittenen Männern könne die Zirkumzision eine sinnvolle Maßnahme zur Senkung der HIVNeuinfektionsrate sein. Bei anderen Infektionen wie Syphilis, Herpes oder Gonorrhoe ist die Datenlage unzureichend. Diese Erkenntnisse sind einerseits nicht auf andere Kontinente übertragbar und andererseits ist die Verwendung von Kondomen weiterhin sicherer zur Vermeidung sexuell übertragbarer Erkrankungen.

Der Einfluss auf die Entstehung maligner Erkrankungen durch eine Phimose ist ebenfalls fraglich. Das Risiko für die Entstehung eines Peniskarzinoms scheint bei beschnittenen Männern reduziert zu sein. Insgesamt ist die Inzidenz des Peniskarzinoms in der westlichen Welt jedoch bereits sehr gering und in den letzten Jahren weiter gesunken. Man schätzt ca. 900 Zirkumzisionen durchführen zu müssen, um ein Peniskarzinom zu vermeiden und gleichzeitig jedoch zwei Komplikationen in Kauf zu nehmen [7].

\section{Rituelle Beschneidung \\ $\nabla$}

Die häufigste Begründung für nicht medizinisch indizierte Zirkumzisionen im Säuglings- und Kindesalter sind religiöse Motive [8]. Insbesondere im Judentum und im Islam hat das Ritual einen besonderen Stellenwert. Während im Judentum die Beschneidung am 8. Tag nach der Geburt durchgeführt werden soll und nur in besonderen Fällen aufgeschoben wird, findet die rituelle Beschneidung im Islam ohne exakt festgelegten Zeitpunkt meistens bis zum Beginn der Pubertät statt und signalisiert die Aufnahme als vollwertiges männliches Mitglied in die Glaubensgemeinschaft. Nachdem die Rechtslage zu den medizinisch nicht indizierten Beschneidungen lange Zeit unklar war, wurde aufgrund einer Gesetzesänderung $§ 1631 \mathrm{~d}$ in das Bürgerliche Gesetzbuch der Bundesrepublik Deutschland eingefügt. Dieser erlaubt es Eltern, wie eingangs bereits dargestellt, bei religiös motivierten Beschneidungen eines nicht einwilligungsfähigen Kindes in die Beschneidung einzuwilligen. Voraussetzung hierfür ist, dass die Beschneidung nach den Regeln der ärztlichen Kunst durchgeführt wird. Im ersten halben Jahr nach der Geburt des Kindes darf die Beschneidung jedoch auch von entsprechend ausgebildeten, nicht ärztlichen Personen der Religionsgesellschaft durchgeführt werden. Spezielle Anforderungen oder die Kriterien für diese Ausbildung werden im Gesetzestext nicht de- 
finiert und den Religionsgesellschaften überlassen. Verlangt wird in diesem Zusammenhang aber dennoch eine besondere Ausbildung. Tatsächlich handelt es sich hierbei um eine Ausnahme vom Arztvorbehalt, die allerdings den „Behandler“ nicht davon befreit, seine Tätigkeit auf dem Niveau des Facharztstandards zu erbringen.

Die medizinische, gesellschaftliche und politische Diskussion während der Entstehung des Gesetzentwurfs entbrannte anhand der vermeintlichen Kollision zweier im deutschen Grundgesetz verankerter Grundrechte: Dem Recht auf körperliche Unversehrtheit des Kindes (Artikel 2, Absatz 2 Grundgesetz) und dem Recht auf ungestörte Religionsausübung der Eltern (Artikel 4, Absatz 2 Grundgesetz).

Hierbei spielte eine Rolle, dass nach wie vor der rechtliche Grundsatz gilt, dass ein operativer Eingriff den Straftatbestand der Körperverletzung erfüllt und dieser nur straffrei ist, wenn in die Behandlung wirksam eingewilligt worden ist. Typischerweise sind die Betroffenen bei der nicht medizinisch indizierten Zirkumzision wegen ihres Alters nicht einwilligungsfähig. Daher muss die Einwilligung des Patienten ersetzt werden. Dies geschieht in der Regel durch die Einholung der Einwilligung der Sorgeberechtigten, mithin also üblicherweise beider Eltern. Deren Rechte ergeben sich aus Artikel6, Absatz 2 des Grundgesetzes, der wiederum seinen Widerhall auch im Bürgerlichen Gesetzbuch findet. Nach den Regelungen des Grundgesetzes und des $§ 1631$ BGB sind die Pflege und Erziehung der Kinder das natürliche Recht der Eltern. Dieses grundrechtlich verbürgte Elternrecht soll primär eine Freiheitssphäre der Eltern für die Kindererziehung gewährleisten, in der die Eltern frei von staatlichem Einfluss ihre Kinder nach eigenen Vorstellungen pflegen und erziehen können und in die der Staat nur eingreifen darf, wenn es gesetzlich erlaubt ist [9]. Allerdings sind die Eltern bei der Ausübung des Sorgerechtes nicht gänzlich frei, sondern haben die Personensorge unter Beachtung des Kindeswohles auszuüben. Dabei gibt die Rechtsprechung den Eltern bei der Frage des Kindeswohls einen großen Freiraum und sieht die Grenzen der Ausübung des Kindeswohls erst dann erreicht, wenn sich bei der weiteren Entwicklung der Dinge eine erhebliche Schädigung des geistigen oder leiblichen Wohls des Kindes mit ziemlicher Sicherheit voraussehen lässt [10]. Neben diesen nationalen familienrechtlichen Regelungen ist in der Bundesrepublik Deutschland bereits seit 1992 die Kinderrechtskonvention der Vereinten Nationen in Kraft, mit der sich in Artikel 24, Absatz 3 die Mitgliedsstaaten verpflichten, alle wirksamen und geeigneten Maßnahmen zu treffen, um überlieferte Bräuche, die für die Gesundheit der Kinder schädlich sind, abzuschaffen.

Ob die Beschneidung dem Kindeswohl entspricht respektive diesem schädlich ist oder nicht, wurde bereits früher in diesem Artikel diskutiert. Die medizinischen Vor- und Nachteile scheinen sich dabei die Waage zu halten. Für die befürwortenden Glaubensgemeinschaften stellt die Durchführung des Eingriffs jedoch ein entscheidendes Ritual dar, mit dem die Glaubenszugehörigkeit ausgedrückt wird. Obwohl sowohl im Islam als auch im Judentum Gegner der Beschneidungspraxis zu finden sind, ist für die meisten Gläubigen das Beschneidungsritual unantastbar. So ist das Argument der identifikationsstiftenden Beschneidung nicht von der Hand zu weisen.

Vor diesem Hintergrund stellt sich schon die Frage, ob die Einwilligung der Eltern zur Zirkumzision nicht schon allein unter Beachtung der medizinischen Kriterien möglich ist, da diese dem Kindeswohl nicht zuwider läuft - der Eingriff ist nach der gezeigten medizinischen Datenlage nicht unvertretbar. Die Bundesre- gierung hat allerdings durch die Ergänzung des „Beschneidungsgesetzes“ Zweifel ausgeräumt und in verfassungsrechtlich wohl unbedenklicher Weise $[11,12]$ Rechtsklarheit für die Zulässigkeit der „Beschneidung“ geschaffen. Dabei hat sie in der Gesetzesergänzung klar die Zulässigkeit der „Beschneidung des männlichen Kindes" auch in nicht medizinisch indizierten Fällen geklärt und bis auf Ausnahmen zugelassen. Tatsächlich wurde so der verfassungsrechtlich geschützte Anspruch auf freie religiöse Entfaltung wiederholt und der Staat in dieser Frage zur Neutralität verpflichtet, soweit es zur Vermeidung von Gefahren für das Kindeswohl nicht notwendig war. Die Entscheidung selbst liegt insoweit bei den Eltern, deren Überzeugungen insgesamt in die Erziehung des Kindes einfließen, wovon auch die religiöse Überzeugung umfasst wird.

Es bleibt allerdings der Zwiespalt, dass der Gesetzgeber zwar insoweit erfolgreich der Aufgabe nachgekommen ist, vielen Interessengemeinschaften in unserem Land gerecht zu werden. Dem Interesse eines Kindes auf einen unversehrten Körper ist er damit hier nicht nachgekommen. Es besteht insoweit ein Konflikt zur klar geregelten Erziehung unter Gewaltverzicht des $§ 1631 \mathrm{Ab}$ satz 2 BGB.

Es bleibt für den Mediziner, an den die Behandlung herangetragen wird, über die fehlende medizinische Indikation aufzuklären und auch auf die Zweifelhaftigkeit der vermeintlichen Vorteile hinzuweisen. Wie bei jeder nicht medizinisch indizierten Behandlung ist besonders deutlich auch auf die Risiken des Eingriffes und der möglichen postoperativen Komplikationen im Rahmen der Aufklärung hinzuweisen. Diese Aufklärung gegenüber beiden Eltern wie auch die Einwilligung beider Eltern muss sorgsam schriftlich dokumentiert werden. Bei Zweifeln gilt es die Behandlung zu versagen. Die freie Ausübung des Elternrechtes in religiösen Fragen hat in der Regel ihr Ende mit dem 12.Lebensjahr. Ab diesem Alter bedarf es der Aufklärung und Einwilligung des Kindes und der Eltern, ab dem 14. Lebensjahr wird regelmäßig das Kind alleine entscheiden. Keinesfalls dürfen die Regelungen zur „Beschneidung des männlichen Kindes“ auf weibliche Kinder angewendet werden. Dies war bereits in der Vergangenheit so und wurde nunmehr zur Klarstellung auch ausdrücklich mit $\S 226$ a StGB neu in das Strafgesetzbuch aufgenommen. Deren Beschneidung ist als verboten anzusehen [13]. Die Behandlung selbst ist nach Facharztstandard auszuführen, was auch für die anästhesiologischen und schmerztherapeutischen Teile der Behandlung sowie für die Nachsorge gilt [12]. Die Behandlung ist zu unterlassen, wenn Erkenntnisse vorliegen, die den Eingriff als im Einzelfall unmittelbar gefährlich ansehen lassen. Sind die Eltern in diese Fällen uneinsichtig und droht dennoch die Behandlung des Kindes, ist der Arzt berechtigt, das zuständige Jugendamt zu informieren ( $\$ 4 K K$ K) [12]. Sieht sich der angestellte Arzt ernsthaften Gewissenskonflikten ausgesetzt, so kann er - unter Offenlegung derselben gegenüber seinem Arbeitgeber - die Tätigkeit auch ablehnen [14]. Für den selbstständigen Arzt gilt dies sowieso.

\section{Interessenkonflikt \\ $\nabla$}

Die Autoren geben an, dass kein Interessenkonflikt besteht. 


\section{Abstract}

\section{Circumcision Might Cause Conflicts Between Fundamental Rights}

$\nabla$

Circumcision of male infants often causes political, social and medical controversial discussions. Different lobbies take part in the debate. Especially religious and social indication for this medical intervention often is discussed to clash with fundamental rights in our society. Our article addresses not only historical and medical aspects of the discussion but highlights the legal background of male circumcision in infants.

\section{Literatur}

1 Campbell MF, Harrison JH. Urology. 3rd ed. Philadelphia: W. B. Saunders, Vol. 2; 1970: 1836

2 Stehr M, Putzke H, Dietz H-G. Zirkumzision bei nicht einwilligungsfähigen Jungen: Strafrechtliche Konsequenzen auch bei religiöser Begründung. Deutsches Ärzteblatt 2008; 105: 34-35

3 LG Köln, 7.5.2012, 151 Ns 169/11
4 American Academy of Pediatrics. Task Force on Circumcision. Male circumcision. Pediatrics 2012; 130: e756-785

5 Kim D, Pang MG. The effect of male circumcision on sexuality. BJU Int 2007; 99: 619-622

6 Morris BJ, Krieger JN. Does Male Circumcision Affect Sexual Function, Sensitivity, or Satisfaction? - A Systematic Review. J Sex Med 2013 Aug 12. doi: 10.1111/jsm.12293 [Epub ahead of print]

7 Christakis DA, Harvey E, Zerr DM et al. A trade-off analysis of routine newborn circumcision. Pediatrics 2000; 105 (1 Pt 3): 246-249

8 Stehr M, Putzke H, Dietz H-G. Zirkumzision bei nicht einwilligungsfähigen Jungen: Strafrechtliche Konsequenzen auch bei religiöser Begründung. Deutsches Ärzteblatt 2008; 105: 34-35

9 Bundesverfassungsgericht 16.1.2003, 2 BvR 716/01. Barbara Veit in Beck'scher Online-Kommentar BGB (Stand 2011), Hrsg. Bamberger/ Roth § 1626 Rn 7-13

10 Bundesgerichtshof ständige Rechtsprechung seit NJW 1956, 1434 , so auch Entscheidung vom 19.Dezember 2004, XII ZB 166/03; BGH 16.10.2011, XII ZB 247/11

11 Germann M. Gesetz über den Umfang der Personensorge bei einer Beschneidung. Medizinrecht 2013: 412-424

12 Rixen S. Das Gesetz über den Umfang der Personensorge bei einer Beschneidung des männlichen Kindes. Neue Juristische Wochenzeitung (NJW) 2013: 257-262

13 Bundestagsdrucksache 17/11295, S. 17

14 Bundesarbeitsgericht, 22.05.2003, 2 AZR 426/02; Urteil vom 24. Mai 1989 - 2 AZR 285/88; Urteil vom 20. Dezember 1984 - 2 AZR 436/83 